\title{
Suramin protects hepatocytes from LPS-induced apoptosis by regulating mitochondrial stress and inactivating the JNK-Mst1 signaling pathway
}

\author{
Aizhong Wang ${ }^{1} \cdot$ Jiali Wang $^{2} \cdot$ Jun $\mathrm{Wu}^{2} \cdot$ Xiaojun Deng $^{2} \cdot$ Yan Zou $^{2}$ \\ Received: 26 November 2018 / Accepted: 3 February 2019 / Published online: 15 February 2019 \\ (c) The Physiological Society of Japan and Springer Japan KK, part of Springer Nature 2019
}

\begin{abstract}
An uncontrolled inflammatory response has been implicated in the progression of acute liver failure through poorly understood mechanisms. The aim of our study was to investigate whether suramin attenuates inflammation-mediated hepatocyte apoptosis by modulating mitochondrial homeostasis. Primary hepatocytes were isolated from mice and treated with LPS in vitro in the presence or absence of suramin. Western blotting, immunofluorescence staining, and ELISAs were used to evaluate the mitochondrial stress. The LPS treatment caused hepatocyte death via apoptosis. Interestingly, suramin supplementation attenuated LPS-mediated hepatocyte death by reducing Mst1 expression; the overexpression of Mst1 abolished the anti-apoptotic effects of suramin on LPS-treated hepatocytes. At the molecular level, suramin treatment repressed mitochondrial oxidative stress, sustained mitochondrial dynamics and blocked the caspase-9-mediated mitochondrial apoptosis pathway; these effects of suramin were achieved by reversing Mst1 expression. Furthermore, our study found that suramin modulated Mst1 expression via the JNK signaling pathway. Activation of JNK prevented the suramin-mediated Mst1 downregulation and concomitantly increased hepatocyte apoptosis and mitochondrial dysfunction. Taken together, our results confirmed the anti-apoptotic and anti-inflammatory effects of suramin on LPS-challenged hepatocytes. Suramin sustained hepatocyte viability and attenuated mitochondrial stress via repressing the JNK-Mst1 signaling pathway.
\end{abstract}

Keywords Suramin $\cdot$ Inflammation $\cdot$ Mitochondria $\cdot$ Hepatocyte death $\cdot$ Mst $1 \cdot$ JNK pathway

\section{Introduction}

Many etiologies are responsible for the development of acute liver failure (ALF), including drug-related acute liver injury, viral hepatitis, ischemia hepatitis, metabolic disorders, and neonatal hemochromatosis. At the molecular level, inflammation-induced injury has been acknowledged as a primary cause of ALF pathogenesis [1]. Excess inflammatory stress induces oxidative stress in the cell and the subsequent activation of the c-Jun N-terminal kinase (JNK) signaling pathway [2]. Activated JNK translocates to the nucleus, where JNK promotes the transcription and expression of genes related to hepatocyte death [3]. Moreover, inflammatory stress also activates hepatic macrophages to potentiate liver injury by releasing pro-inflammatory factors. Subsequently, with the help of pro-inflammatory cytokines, immune cells such as neutrophils and monocytes are recruited to the site of inflammation. Excess neutrophil activation assists in the removal of necrotic hepatocytes, followed by a loss of functional 
hepatocytes in a short period [4]. Anti-inflammatory therapy was shown to be beneficial for damaged liver tissues in animal studies and human clinical trials $[5,6]$.

Recently, the mitochondrion has been reported to be the downstream target of inflammatory injury. Substantial accumulation of swollen mitochondria has been observed in patients with ALF, suggesting that inflammatory stress seems to regulate mitochondrial morphology. Additionally, a reduction in the mitochondrial membrane potential [7], mitochondrial Bax-induced apoptosis [8], mitochondrial respiratory dysfunction [9], mitochondrial biogenesis arrest [10], and mitochondrial oxidative stress [11] have been implicated in the development of ALF due to the uncontrolled inflammation-induced injury. Based on this evidence, the mitochondria may have the ability to favorably modulate hepatocyte function and viability during inflammation-induced liver injury. However, no study has provided insights into the basic mechanisms of mitochondrial stress in the setting of inflammation-induced hepatocyte death.

Suramin has been widely used to treat trypanosomiasis and recently has been shown to regulate the inflammation response in various disease models. For example, suramin treatment attenuates inflammation-mediated skin damage in atopic dermatitis [12]. Besides, suramin also inhibits inflammation-related peritoneal fibrosis [13]. Moreover, suramin has been found to be effective for diabetic nephropathy [14]. In LPS-treated alveolar epithelial cells, suramin has an ability to inhibit the expression and transcription of inducible nitric oxide synthase. More importantly, the beneficial effects of suramin on hepatic dysfunction have been reported. Suramin improves the regeneration of ethanolinduced steatotic partial liver grafts [15]. Besides, the function and stress response of liver macrophages/Kupffer cells are also modulated by suramin. However, the exact mechanisms by which suramin confers protection, and the key cellular parameters that it influences in inflammation-mediated hepatocyte mitochondrial death, remain to be elucidated.

At the molecular level, mammalian Ste20-like kinase 1 (Mst1) is an apoptosis-related kinase that preferentially modulates the mitochondria-involved apoptosis signal in liver tissue. In the setting of liver cancer, activated Mst1 promotes cancer cell apoptosis through a mechanism depending on mitochondrial apoptosis [16]. Also, pharmacological targeting of kinase Mst1 augments liver tissue repair and regeneration [17]. Moreover, hepatic lipid metabolism is also regulated by Mst1 via repressing the activity of Sirt1 [18]. In the present study, we wanted to determine whether Mst1 is activated by LPS and contributes to the progression of inflammation-related liver injury via inducing mitochondrial stress. Meanwhile, we also explored whether suramin had an ability to confer protection against inflammation-induced hepatocyte death via regulating Mst1 and mitochondrial homeostasis.

\section{Materials and methods}

\section{Isolation and culture of primary hepatocytes}

Primary hepatocytes were isolated using methods reported in a previous study. C57/B16 mice (8-10 weeks old, female) were anesthetized using a mixture of ketamine $(80 \mathrm{mg} / \mathrm{kg})$ and xylazine $(5 \mathrm{mg} / \mathrm{kg}$ ). Then, a catheter ( $22 \mathrm{G}$ feeding needle/round tip, connected to Solution 1) was inserted into the vena cava through the right atrium. Subsequently, perfusion was started (flow rate of $5 \mathrm{ml} / \mathrm{min}$ ) [19]. After the flow began and the buffer reached the liver, a small incision in the visceral vena cava was made. After collagenase perfusion, liver should begin to look mushy. Dissect the liver free, place in a pre-chilled sterile beaker with $20 \mathrm{ml}$ William's complete Medium, and then take it to tissue cell culture hood. Within the cell culture hood, use a cell scraper to gently disperse the cells into William's complete Medium within a sterile Petri dish. Finally, after washing the pellet twice with PBS, primary hepatocytes were collected and re-suspended in DMEM supplemented with 20\% FBS. Cells were treated with $10 \mu \mathrm{g} / \mathrm{ml}$ LPS (Selleck Chemicals) for approximately $12 \mathrm{~h}$ to induce inflammatory injury. Suramin $(0-20 \mu \mathrm{M})$ was also incubated with hepatocytes for $12 \mathrm{~h}$ during the LPS challenge. Anisomycin (Ani, $10 \mu \mathrm{M}$, Selleck Chemicals, Houston, TX, USA) was added to the medium of cultured hepatocytes and incubated for $2 \mathrm{~h}$ to activate the JNK signaling pathway. The Mst1 adenovirus was transfected into suramin-treated hepatocytes to reverse Mst1 expression [20].

\section{Cellular death was measured via a TUNEL assay}

TUNEL staining was performed using a One Step TUNEL Apoptosis Assay Kit (Beyotime, China, Cat. No: C1086) according to the manufacturer's instructions [21]. The MTT assay was performed according to the methods used in a previous study. Cells were plated onto a 96-well plate. MTT solution (Beyotime, China, Cat. No: C0009) was then added into the medium, and the cells were incubated for approximately $2 \mathrm{~h}$ at $37{ }^{\circ} \mathrm{C} / 5 \% \mathrm{CO}_{2}$. The optical density (OD) of the MTT solution was recorded using a microplate reader (490 nm absorbance; Epoch 2; BioTek Instruments, Inc., Winooski, VT, USA) [22]. An LDH release assay was conducted using a commercial kit (Beyotime, China, Cat. No: C0016) according to the manufacturer's instructions [23].

\section{Caspase activity detection and ELISA}

The caspase- 3 and caspase- 9 activities were determined using commercial kits (Beyotime Institute of Biotechnology) [24]. The levels of antioxidant factors, including GPX, SOD, 
and GSH, were measured with ELISA kits purchased from the Beyotime Institute of Biotechnology [25]. The experiments were performed in triplicate and repeated 3 times with similar results.

\section{Mitochondrial potential observation and LDH release assay}

The mitochondrial membrane potential was determined by JC-1 staining. Live cells were washed with PBS, and a JC-1 solution was then added to the medium [26]. The cells were incubated at $37{ }^{\circ} \mathrm{C} / 5 \% \mathrm{CO}_{2}$ for $30 \mathrm{~min}$, washed with PBS, loaded with DAPI, and then observed under a fluorescence microscope. An LDH release assay was conducted using a commercial kit (Beyotime, China, Cat. No: C0016) according to the manufacturer's instructions [27].

\section{ATP detection}

Cells were seeded in six-well plates at $1 \times 106$ cells per well, and the cells were then incubated at $37{ }^{\circ} \mathrm{C}$ in $5 \% \mathrm{CO}_{2}$ until $80 \%$ confluent. The intracellular concentration of ATP was analyzed using an ATP Assay Kit (Beyotime, China) [28]. To each well, $200 \mu \mathrm{l}$ lysate was added. The supernatant was collected after centrifugation for $5 \mathrm{~min}$ at $12,000 \mathrm{~g}$ and $4{ }^{\circ} \mathrm{C}$. The intracellular concentration of ATP was measured using a luminometer according to the protocol provided by Beyotime [29].

\section{Immunofluorescence staining}

Cells were washed with PBS at room temperature to remove the DMEM. Then, the cells were fixed in 3.7\% paraformaldehyde for $30 \mathrm{~min}$ at room temperature and permeabilized with $0.1 \%$ Triton $\mathrm{X}-100$ for $10 \mathrm{~min}$ at $4{ }^{\circ} \mathrm{C}$. The cells were then washed with PBS, and $10 \%$ goat serum albumin was used to block the samples for $45 \mathrm{~min}$ at room temperature [30]. The samples were again washed with PBS and then incubated overnight with the following primary antibodies: Tom20 (1:1000, Abcam, \#ab186735), p-JNK (1:1000, Abcam, \#ab81283), Mst1 (1:1000, Abcam, no. ab86671).

\section{Determination of GSH, SOD and GPx levels}

The GSH content and the SOD and GPx activity levels in LPS-challenged hepatocytes were determined using commercially available GSH, SOD and GPx kits, according to the manufacturer's instructions [31]. The data were analyzed spectrophotometrically using a SpectraMax M5 instrument (Molecular Devices, CA, USA).

\section{Flow cytometry for detecting mitochondrial ROS levels}

Mitochondrial ROS production was measured using flow cytometry. Briefly, $1 \times 10^{6}$ hepatocytes were labeled with MitoSOX red mitochondrial superoxide indicator (Molecular Probes, USA) for $15 \mathrm{~min}$ at $37^{\circ} \mathrm{C}$. Labeled cells were washed twice with culture medium and then harvested [32]. The resulting fluorescence was measured using flow cytometry.

\section{Western blot}

Cells were lysed in RIPA Lysis Buffer (Beyotime, China, Cat. No: P0013C). After high-speed centrifugation, the proteins were collected and quantified with the Enhanced BCA Protein Assay Kit (Beyotime, China, Cat. No: P0009). Subsequently, 40-60 $\mu \mathrm{g}$ of protein was loaded onto $10 \%$ SDS-PAGE gels and transferred to PVDF membranes. The membranes were washed with TBST and then blocked with 5\% non-fat milk for $45 \mathrm{~min}$ at room temperature [33]. The primary antibodies used in the present study were as follows: Bcl2 (1:1000, Cell Signaling Technology, \#3498), Bax (1:1000, Cell Signaling Technology, \#2772), caspase9 (1:1000, Cell Signaling Technology, \#9504), survivin (1:1000, Cell Signaling Technology, \#2808), Parkin (1:1000, Cell Signaling Technology, Inc.), Beclin1 (1:1000, Cell Signaling Technology, \#3738), Drp1 (1:1000, Abcam, \#ab56788), Fis1 (1:1000, Abcam, \#ab71498), Opa1 (1:1000, Abcam, \#ab42364), Mfn1 (1:1000, Abcam, \#ab57602), Mfn2 (1:1000, Abcam, \#ab56889), Mff (1:1000, Cell Signaling Technology, \#86668), t-JNK (1:1000; Cell Signaling Technology, \#4672), p-JNK (1:1000; Cell Signaling Technology, \#9251), Mst1 (1:1000, Cell Signaling Technology, \#3682).

\section{RNA isolation and quantitative RT-PCR (qPCR)}

After treatment, cellular RNA was isolated via Trizol reagent (Invitrogen, Carlsbad, CA) according to the manufacturer's instructions. Reverse transcription of RNA was performed with a Transcriptor First Strand cDNA Synthesis Kit (Roche Applied Science, Switzerland) [34]. About $1 \mu \mathrm{g}$ of RNA was reversely transcribed into cDNA for each sample. Synthesized cDNA was amplified on the LightCycler ${ }^{\circledR} 480$ System with SYBR Green I Master (Roche Applied Science, Mannheim, Germany). The cycling conditions were as follows: $95{ }^{\circ} \mathrm{C}$ for $10 \mathrm{~min}$, followed by 40 cycles of $95^{\circ} \mathrm{C}$ for $15 \mathrm{~s}$, and $72{ }^{\circ} \mathrm{C}$ for $35 \mathrm{~s}$, for telomere PCR [35]. The experiments were repeated 3 times with triplicates of each sample. Fold change of mRNA expression was normalized by GAPDH as an internal control. The primers used in the present study were as follows: Mst1, the forward primer 5'-GCTTCTGAC 
TCAATGCTTAG- 3 ', and the reverse primer 5'-CCACAT CCTCCTGCCAAG-3'.

\section{Cell transfection}

Cells were seeded in the 6-well plates at a density of $5 \times 10^{5}$ cells per well. The Mst1 adenovirus was obtained from OriGene Technologies, Inc. Mst1 adenovirus infection was performed according to a previous study [36, 37]. In brief, hepatocytes were cultured when the cell confluence reached $70-80 \%$. Then, $200 \mu \mathrm{l}$ of medium containing $75 \mathrm{pmol}$ of Mst 1 adenovirus and $200 \mu \mathrm{l}$ of medium containing $7.5 \mu \mathrm{l}$ of Lipofectamine 2000 (Invitrogen, Carlsbad, CA, USA) were mixed together. Subsequently, cells were incubated with the mixture for $48 \mathrm{~h}$. Western blotting was used to detect the overexpression efficiency [38].

\section{Statistical analysis}

We used Statistical Product and Service Solutions (SPSS) 17.0 software (IBM, Armonk, NY, USA) for statistical analyses. The experiments were repeated 3 times $(n=3)$ with triplicates of each sample. The quantitative data are presented as means \pm standard deviations. Differences among groups were analyzed with one-way ANOVA, followed by a post hoc test (least significant difference). Multiple averages among groups were compared using the SNK analysis. $p<0.05$ was considered statistically significant.

\section{Results}

\section{Mst 1 is activated by suramin and promotes hepatocyte survival in the context of LPS-mediated hepatic injury}

In the present study, LPS was used to mimic the inflammatory injury. Then, suramin was administered to detect its effects on LPS-treated hepatocytes. With the help of western blotting, it could be seen that Mst1 expression was significantly upregulated in response to the LPS treatment (Fig. 1a, b). Interestingly, the suramin treatment repressed the changes in Mst 1 expression in a dose-dependent manner (Fig. 1a, b). Subsequently, cell viability was measured using an MTT assay. Compared to the control group, the LPS treatment reduced hepatocyte viability (Fig. 1c), a change that was reversed by the suramin treatment in a dosedependent manner. The minimal protective concentration of suramin was $10 \mu \mathrm{M}$, and thus this concentration was used in the subsequent experiments to perform the functional studies. Moreover, Mst1 adenovirus (Ad-Mst1) was administered to suramin-treated hepatocytes and the overexpression efficiency was confirmed via western blotting to determine whether Mst1 was involved in suramin-mediated cell protection (Fig. 1d, e). Then, cell death was determined using an LDH release assay. As shown in Fig. 1f, compared to the control group, the LPS treatment evoked more LDH release into the medium. However, the suramin treatment inhibited LPS-mediated LDH release, and this effect was abolished by Ad-Mst1 (Fig. 1f). Based on these data, suramin may attenuate LPS-induced hepatocyte death via inhibiting Mst1. This finding was further supported by the TUNEL staining. As shown in Fig. 1g, h, the number of TUNEL-positive cells was obviously increased in response to the LPS treatment. However, the suramin treatment reduced the percentage of TUNEL-positive cells, and this effect was achieved via Mst1 repression because Mst1 overexpression neutralized the anti-apoptotic effects of suramin on LPS-treated cells. Caspase-3 activity was also increased upon LPS challenge (Fig. 1i). However, the suramin treatment inhibited LPSmediated caspase- 3 activation, and this effect was abrogated by the Ad-Mst1 (Fig. 1i). Thus, LPS causes acute hepatocyte death by activating Mst1, and suramin produces prosurvival signals in LPS-treated hepatocytes by preventing Mst1 activation.

\section{Suramin sustains mitochondrial bioenergetics via downregulating Mst 1 in the setting of LPS-induced liver damage}

Mitochondria have been acknowledged as a primary target of LPS-induced inflammatory injury [39]. In the present study, experiments were performed to observe the alterations in mitochondrial function and structure. Firstly, mitochondrial bioenergetics were measured. Total ATP production was reduced by the LPS treatment (Fig. 2a), and this effect was reversed by the suramin treatment. However, after Mst1 overexpression, the effects of suramin on inducing ATP production were repressed (Fig. 2a), indicating that suramin reversed hepatocyte energy metabolism via inhibiting Mst1. Furthermore, cellular ATP is mainly generated by the mitochondria through the conversion of the mitochondrial membrane potential into chemical energy [40]. Interestingly, the mitochondrial membrane potential was significantly decreased by the LPS treatment (Fig. 2b, c), as assessed using JC-1 staining. However, the suramin treatment sustained the mitochondrial membrane potential (Fig. 2b, c), and this effect was nullified by overexpressing Mst1. At the molecular level, mitochondria decompose glucose and form the mitochondrial membrane potential with the help of the mitochondrial respiratory complex. Interestingly, the activity of the mitochondrial respiratory complex was drastically decreased in response to the LPS treatment (Fig. $2 \mathrm{~d}-\mathrm{g}$ ). However, the suramin treatment sustained mitochondrial respiratory complex activity via repressing Mst1 expression (Fig. 2d-g). In addition to 


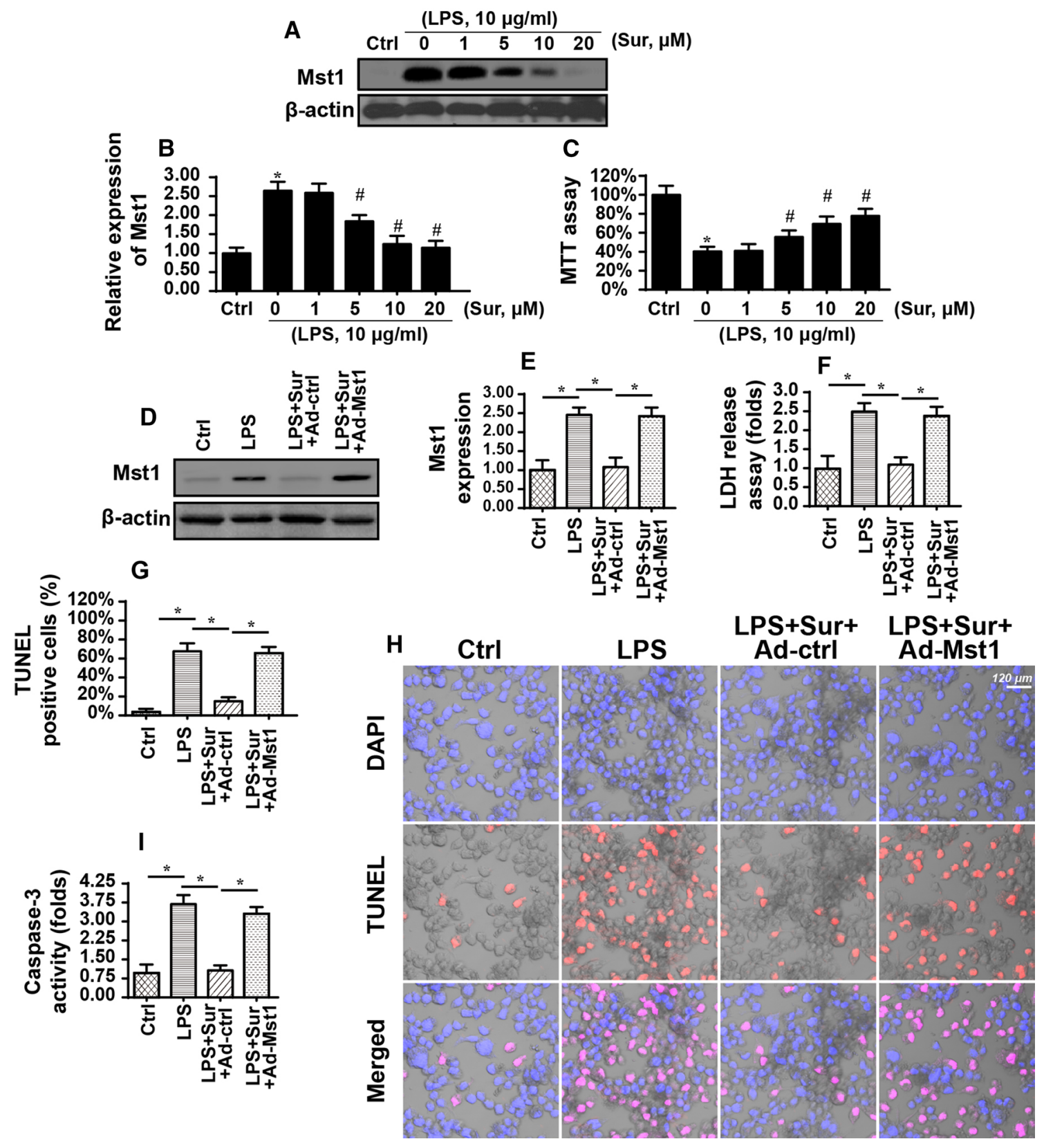

Fig. 1 Suramin attenuates LPS-mediated hepatocyte death by reducing Mst1 expression. a, b LPS was incubated with hepatocytes for $12 \mathrm{~h}$, and different doses of suramin were concomitantly administered to hepatocytes. Then, western blotting was used to observe Mst1 levels. c Cell viability was determined using the MTT assay following the LPS treatment and suramin application. d, e The Mst1 adenovirus (Ad-Mst1) was transfected into hepatocytes, and then Mst1 levels were measured using western blotting. f An LDH release assay

mitochondrial respiratory dysfunction, we also observed increased levels of glucose in the medium upon exposure to LPS (Fig. 2h), indicating that LPS impaired glucose metabolism in hepatocytes. Interestingly, the suramin treatment promoted glucose metabolism in LPS-treated hepatocytes (Fig. 2h), and this effect was abolished by was used to observe cell death in response to suramin in cells with or without Mst1 overexpression. $\mathbf{g}, \mathbf{h}$ TUNEL staining was used to detect apoptotic hepatocytes. The number of TUNEL-positive cells was observed following the suramin treatment and/or Mst1 overexpression. i Caspase-3 activity assay. Hepatocytes were treated with LPS and/or suramin. Meanwhile, Mst1 adenovirus (Ad-Mst1) was used to reverse Mst1 expression. Ad-Mst1 Mst1 adenovirus, Ad-ctrl control adenovirus, ${ }^{*} p<0.05$

Ad-Mst1 transfection. Moreover, lactic acid production was also decreased by the LPS treatment (Fig. 2i), and this effect was reversed by the suramin treatment in a manner dependent on Mst1 repression (Fig. 2i). Based on these results, suramin improves mitochondrial bioenergetics in LPS-treated hepatocytes by inhibiting Mst1. 

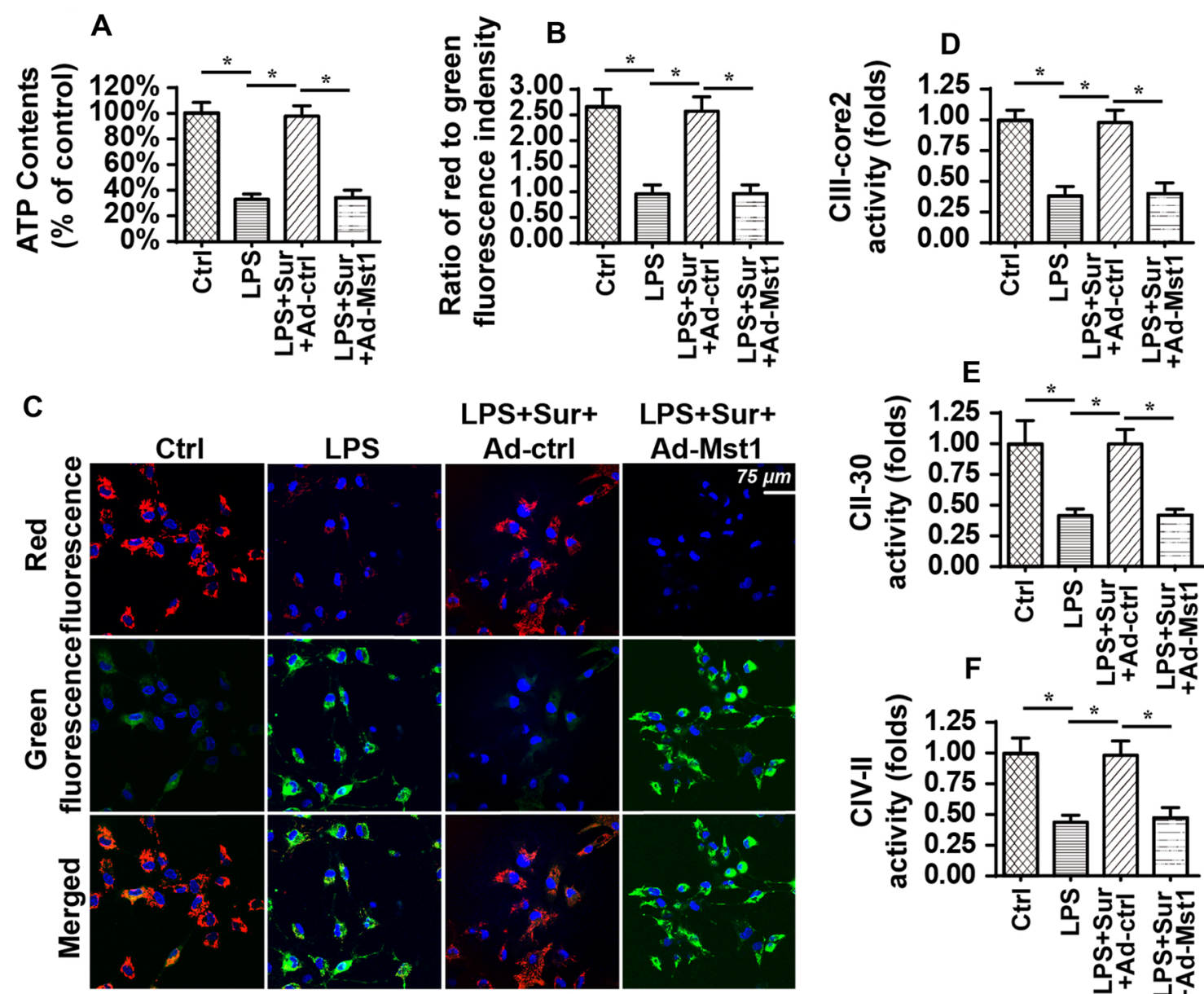

LPS+Sur+ LPS+Sur+
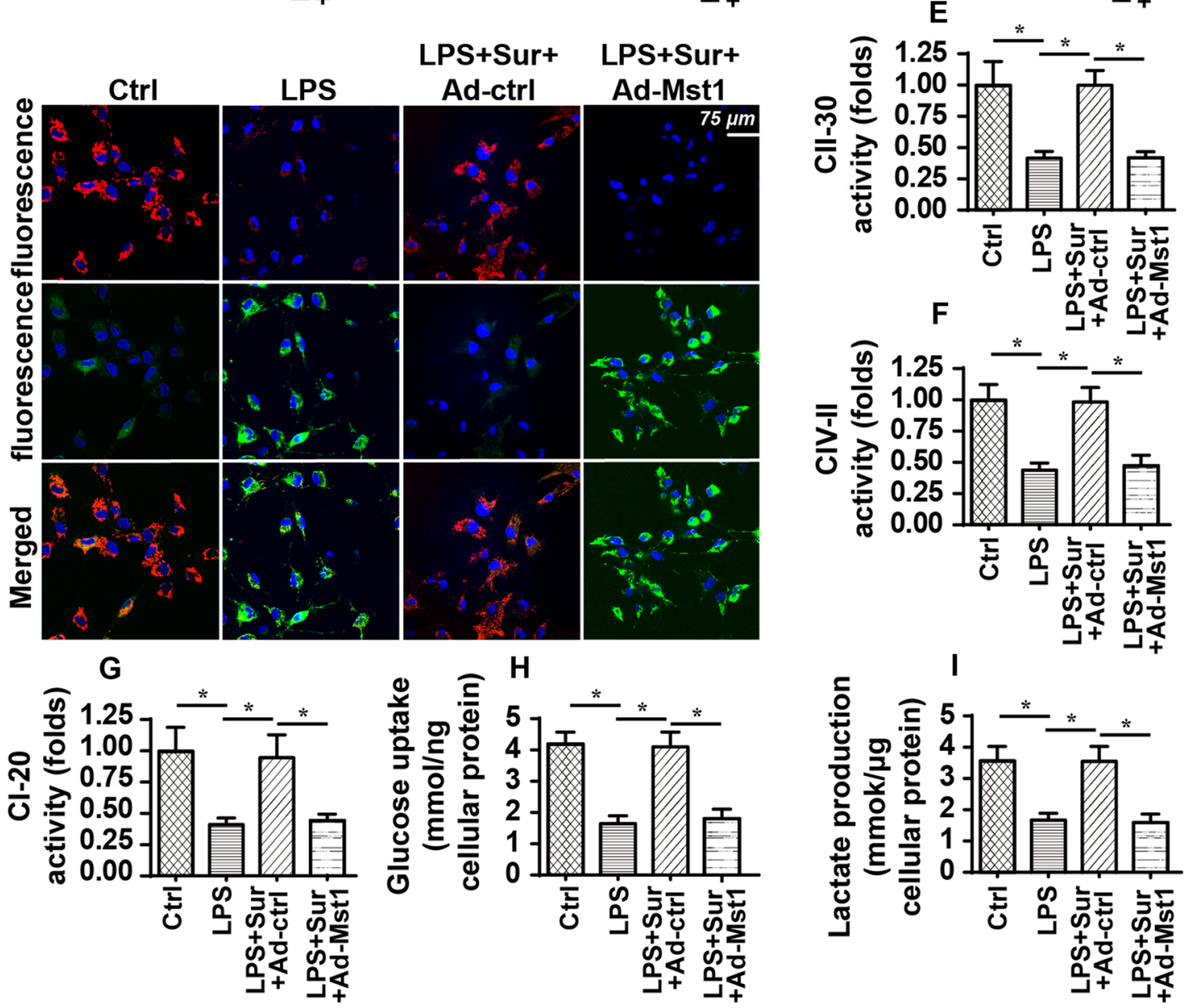

Fig. 2 Mitochondrial bioenergetics are regulated by suramin via repressing Mst1 expression. a ATP production was measured to reflect cellular metabolism in the setting of LPS-induced inflammation. b, c The mitochondrial membrane potential was determined using the JC-1 probe. The relative mitochondrial membrane potential was calculated by measuring the ratio of red/green fluorescence

\section{Mst1 participates in suramin-mediated regulation of mitochondrial dynamics}

Recently, changes in mitochondrial dynamics have been reported to be an early event in mitochondrial stress [41, 42]. Excess mitochondrial fission and decreased mitochondrial fusion promote the formation of mitochondrial fragments, intensities. d-g ELISA was used to observe the alterations in components of the mitochondrial respiratory complex in response to LPS treatment. h Glucose uptake assay using hepatocytes challenged with LPS. i Lactic acid production was evaluated using an ELISA. AdMst 1 Mst 1 adenovirus, Ad-ctrl control adenovirus, ${ }^{*} p<0.05$

which contribute to mitochondrial dysfunction and cell death in several disease models $[8,43]$. In the present study, we explored the effects of suramin on mitochondrial dynamics in the setting of LPS-induced hepatic inflammation damage. With the help of western blotting, it could be seen that the levels of factors related to mitochondrial fission were rapidly increased in response to the LPS treatment (Fig. 3a-d). 

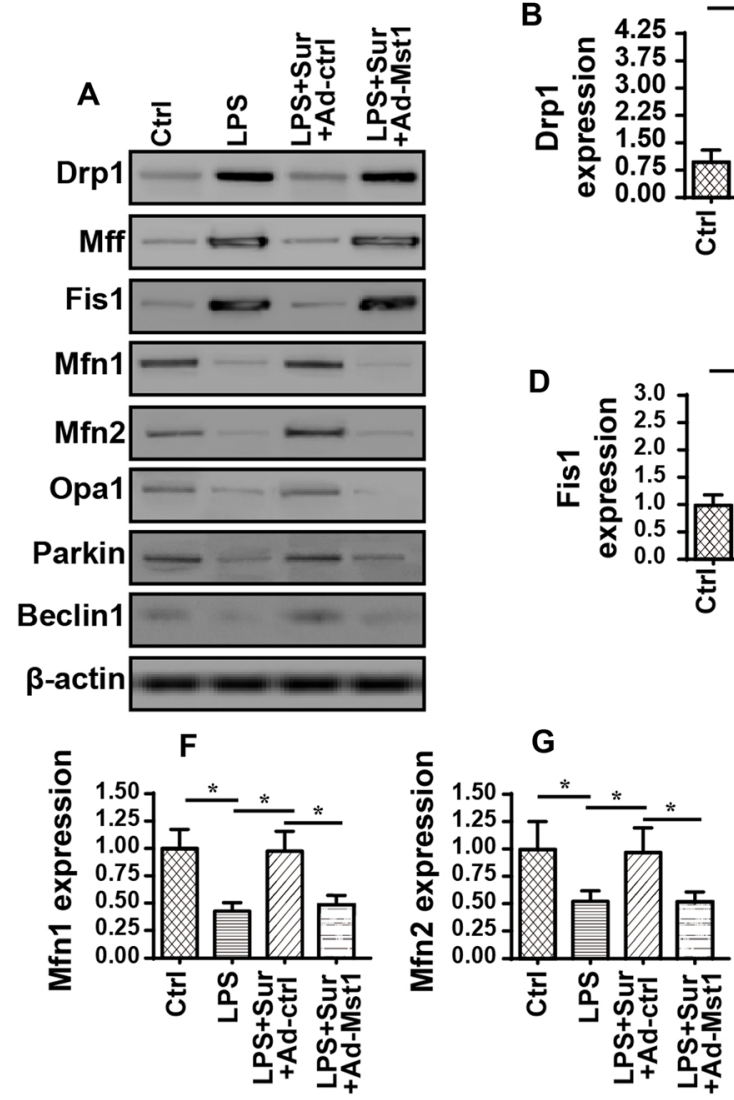

B

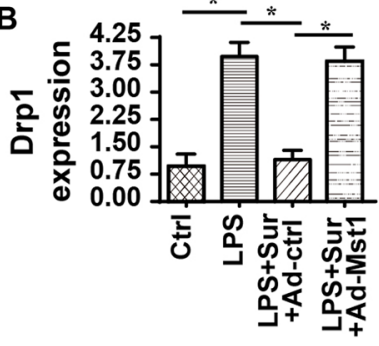

D
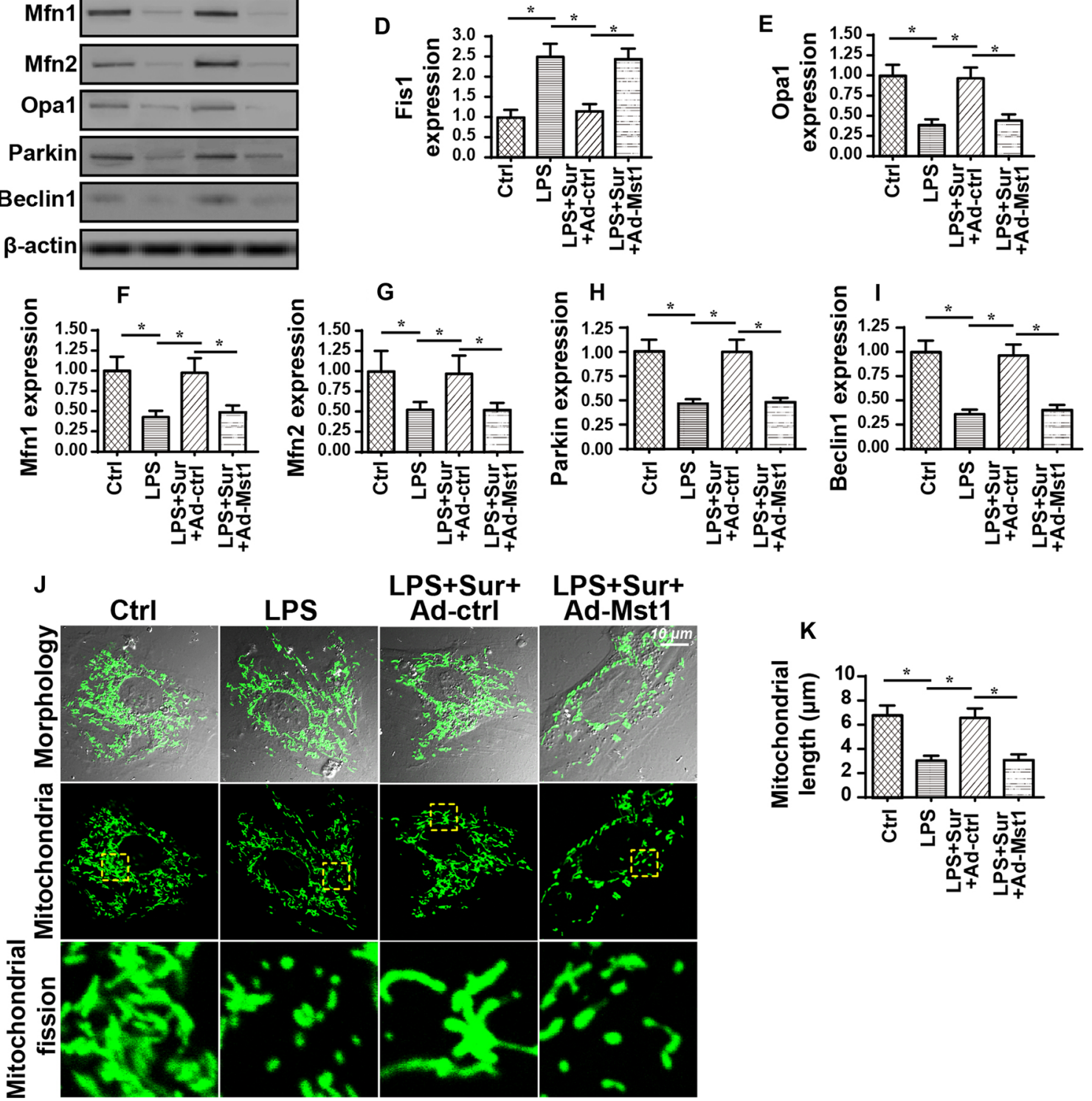

C

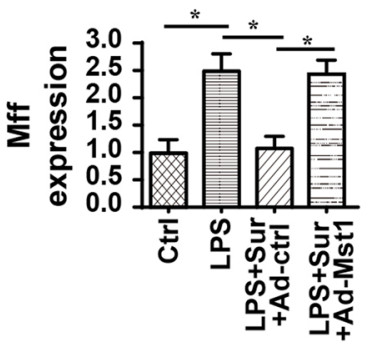

E

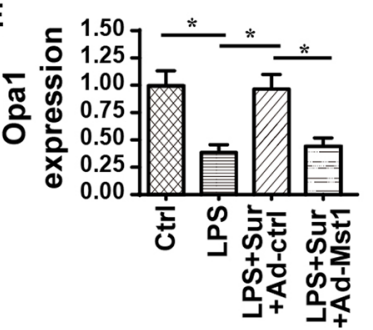


Interestingly, the expression of mitochondrial fusion factors, such as Mfn2, Mfn1 and OPA1, were significantly decreased in response to the LPS stimulus. Thus, inflammation causes an imbalance between mitochondrial fission and mitochondrial fusion (Fig. 3a-g). Additionally, the levels of markers related to mitochondrial autophagy, such as Parkin and Beclin1 (Fig. 3a-i), were also decreased by the LPS treatment. Interestingly, the suramin treatment reversed the changes in the parameters related to mitochondrial fission and the levels of mitochondrial fusion factors (Fig. 3a-i). Moreover, the levels of mitophagy markers were also improved by the suramin treatment (Fig. 3a-i). According to these data, suramin stabilizes mitochondrial dynamics in the setting of LPS-related inflammatory injury in hepatocytes.

Subsequently, immunofluorescence staining was conducted to observe the alterations in mitochondrial dynamics using a mitochondria-specific antibody (Tom-20) [44]. As shown in Fig. 3j, k, mitochondria were inter-connective in the control group and displayed a length of $\sim 6.6 \mu \mathrm{m}$. Interestingly, after treatment with LPS, fragmented mitochondria with shorter lengths were noted in hepatocytes, possibly due to the induction of mitochondrial fission, suppression of mitochondrial fusion and induction of mitophagy arrest. However, the suramin treatment reversed the changes in mitochondrial morphology, and this effect was abolished by Mst1 overexpression (Fig. 3j, k). Overall, LPS-induced inflammatory injury disrupts mitochondrial dynamics in hepatocytes, and this effect is relieved by suramin via Mst1 repression.

\section{Suramin blocks LPS-mediated hepatocyte apoptosis by inactivating Mst 1}

Subsequently, we wanted to determine whether mitochondrial apoptosis was also modulated by suramin via the Mst1 pathway in the setting of LPS-mediated inflammatory injury in hepatocytes. Firstly, western blotting revealed substantial increases in the levels of pro-apoptotic proteins related to mitochondrial death, such as Bax and capsase-9, following the LPS treatment (Fig. 4a-e). Interestingly, the levels of anti-apoptotic proteins were correspondingly decreased after exposure to the LPS stimulus (Fig. 4a-e). However, the suramin treatment prevented the increase in the levels of pro-apoptotic factors and decrease in the levels of anti-apoptotic proteins in LPS-treated hepatocytes (Fig. 4a-e). Notably, Mst1 overexpression abolished the effects of suramin on increasing the levels of anti-apoptotic proteins. Thus, suramin has the ability to repress LPS-mediated hepatocyte apoptosis in a manner dependent on Mst1 repression. Additionally, an early feature of mitochondria-mediated cell death is mitochondrial ROS overproduction [45]. Interestingly, using flow cytometry, we found that LPS-mediated mitochondrial
ROS overloading was attenuated by suramin (Fig. 4f, g). Interestingly, Mst1 overexpression induced mitochondrial ROS overproduction in cells treated with suramin (Fig. 4f, g). In response to mitochondrial ROS overloading, the levels of cellular antioxidants were downregulated by LPS treatment and were reversed to near-normal levels with suramin in a manner dependent on Mst1 repression (Fig. 4h-j), indicating that the anti-oxidant property of suramin seems to rely on Mst1 inhibition in the setting of LPS-mediated inflammatory injury in hepatocytes. Moreover, the late feature of the mitochondrial apoptosis pathway, cyt-c translocation into the cytoplasm/nucleus (Fig. 4k, 1), was also noted in LPS-treated hepatocytes. Interestingly, this phenotypic alteration was reversed by the suramin treatment (Fig. 4k, 1). However, Mst 1 overexpression induced cyt-c transport into the nucleus in cells treated with suramin. Taken together, these data confirmed the functional importance of suramin in inhibiting the LPS-initiated mitochondrial apoptosis pathway by preventing Mst1 upregulation.

\section{Suramin affects Mst1 via the JNK pathway}

The next experiment was conducted to verify the mechanism by which suramin regulated Mst1 expression. JNK is the upstream mediator responsible for inducing Mst1 expression. Inactive JNK is associated with Mst1 downregulation in subjects with myocardial reperfusion injury [46] and colon cancer [47]. In this study, we wanted to determine whether the JNK pathway was required for suraminmediated Mst1 repression. Western blotting revealed that the LPS treatment activated the JNK signaling pathway, as evidenced by increased p-JNK levels (Fig. 5a-c). However, the suramin treatment reduced p-JNK levels, an effect that was accompanied by a decrease in Mst1 expression. Interestingly, activation of the JNK signaling pathway with anisomycin (Ani) not only increased p-JNK levels but also augmented Mst1 expression in suramin-treated hepatocytes (Fig. 5a-c). This finding was further supported by the qPCR assay (Supplemental Figure). Besides, immunofluorescence staining was also applied to observe the levels of p-JNK and Mst1. As shown in Fig. 5d-f, little p-JNK and Mst1 expression was noted in the control group. However, the LPS treatment enhanced the fluorescence intensities of p-JNK and Mst1 (Fig. 5d-f), and this effect was reversed by suramin. Notably, activation of the JNK signaling pathway with Ani reversed Mst1 expression. Interestingly, in the absence of LPS stress, anisomycin (Ani) also elevated the levels of Mst1 (Supplemental Figure). Taken together, the JNK pathway is involved in LPS-mediated Mst1 stabilization, and this effect could be inhibited by suramin. 

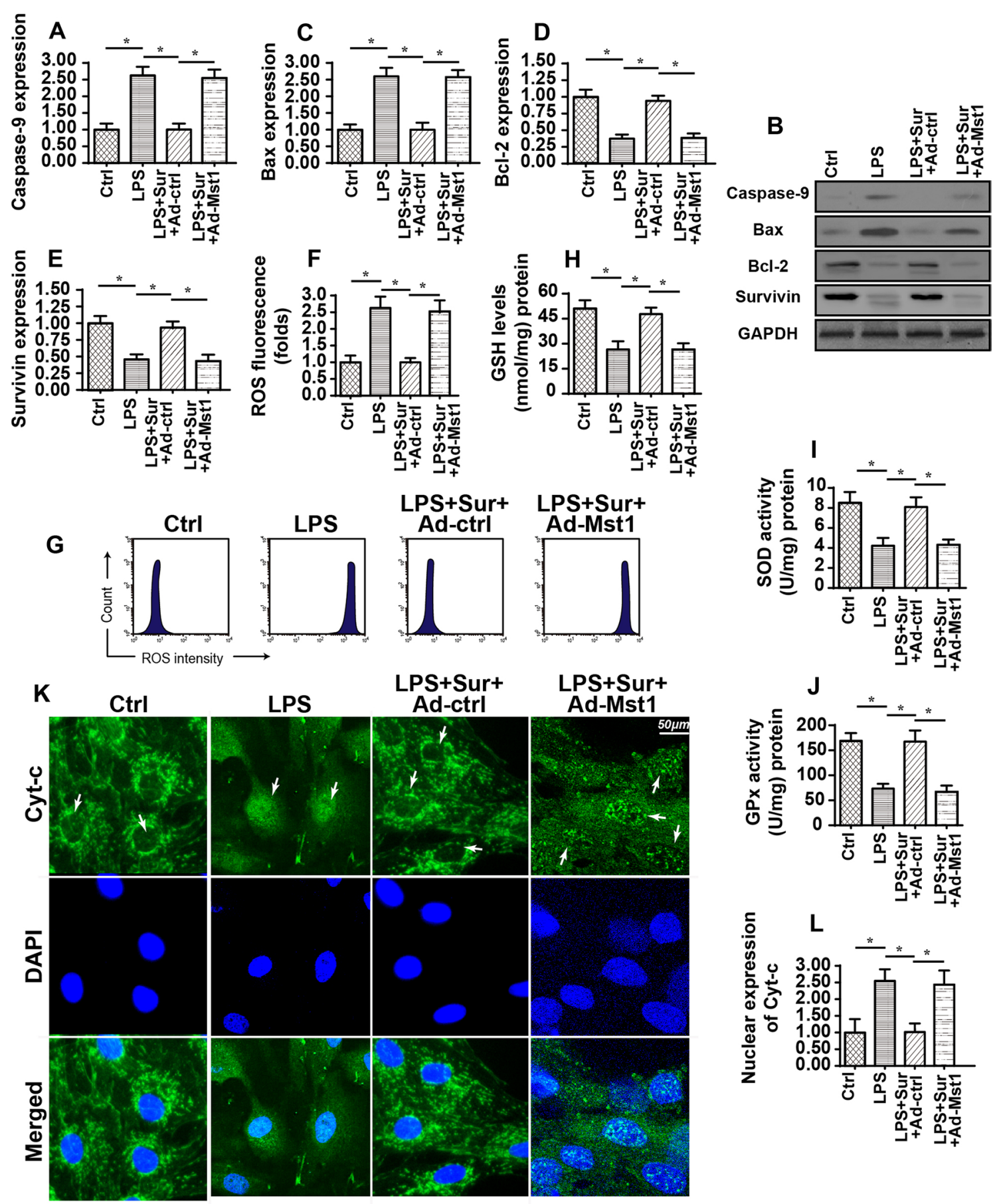

Fig. 4 LPS-induced hepatocyte apoptosis is inhibited by suramin by reducing Mst1 expression. a-e Western blotting was used to detect the alterations in the levels of mitochondrial apoptotic proteins. Hepatocytes were treated with LPS and/or suramin. Meanwhile, Mst1 adenovirus (Ad-Mst1) was used to reverse Mst1 expression. $\mathbf{f}, \mathbf{g}$ Mitochondrial ROS production was determined using flow cytometry. $\mathbf{h}-\mathbf{j}$ The concentrations of cellular antioxidants were measured using ELISAs. Hepatocytes were treated with LPS and/or suramin. Mean- while, Mst1 adenovirus (Ad-Mst1) was used to reverse Mst1 expression. $\mathbf{k}$, I Immunofluorescence staining for liberated cyt-c. DAPI was used to label the nucleus, and the nuclear cyt-c expression was measured to reflect the cyt-c liberation into the cytoplasm/nucleus. Hepatocytes were treated with LPS and/or suramin. Meanwhile, Mst1 adenovirus (Ad-Mst1) was used to reverse Mst1 expression. Ad-Mst1 Mst1 adenovirus, $A d$-ctrl control adenovirus $* p<0.05$ 
Fig. 5 Suramin modulates Mst1 via the JNK signaling pathway. a-c Western blotting was used to observe Mst1 and JNK levels following the LPS and suramin treatments. Meanwhile, anisomycin (Ani) was used to induce JNK activation, after which Mst1 levels were measured. $\mathbf{d}-\mathbf{f}$ Immunofluorescence staining for JNK and Mst1. The relative fluorescence intensity was measured in hepatocytes treated with Ani. $* p<0.05$
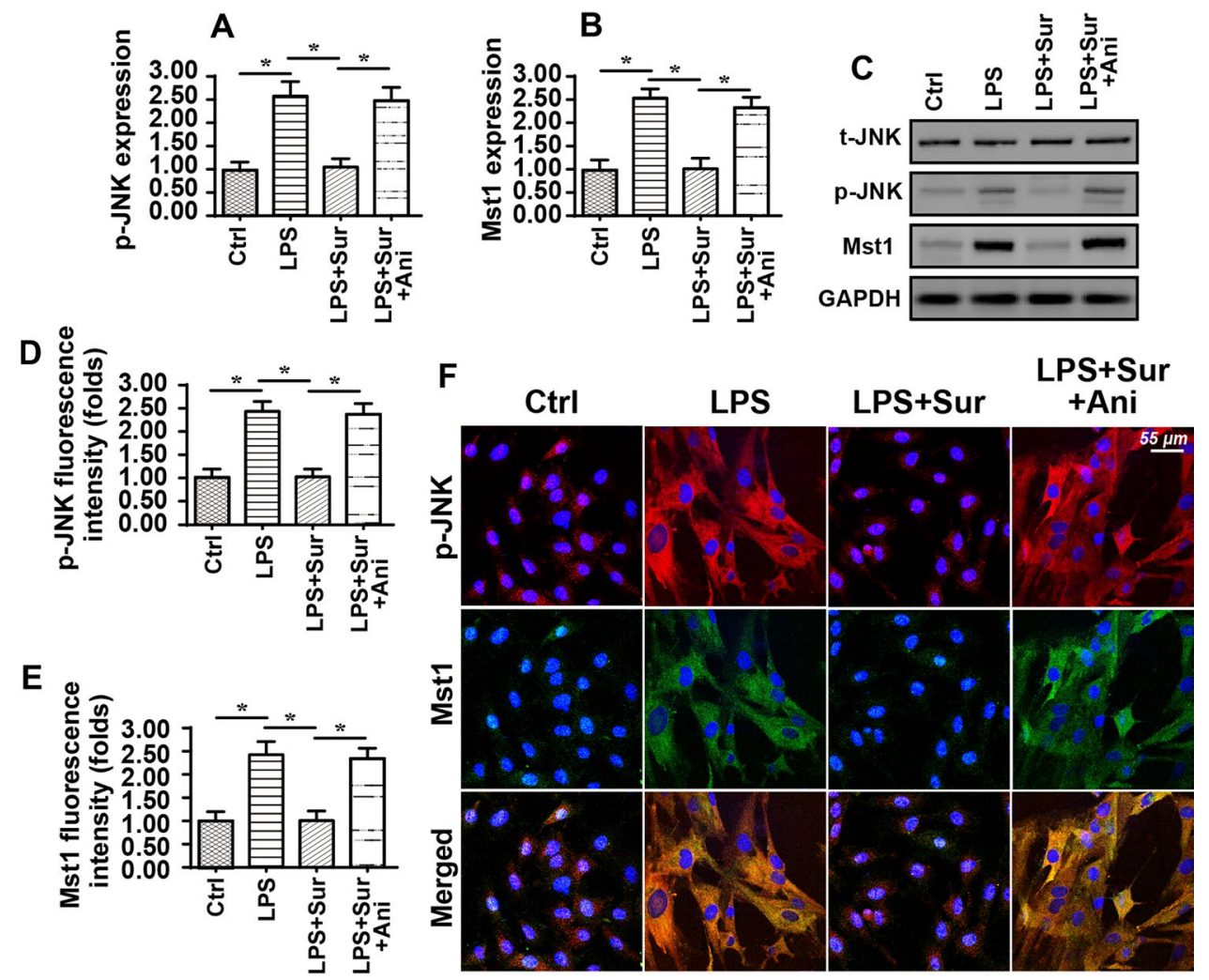

\section{Activation of the JNK signaling pathway abolishes the beneficial effects of suramin on hepatocytes and mitochondria}

Finally, we wanted to investigate whether the JNK pathway was also associated with mitochondrial stress and hepatocyte death. Mitochondrial function was measured in cells treated with Ani to address this question. Mitochondrial oxidative stress, as assessed by flow cytometry, was increased in response to the LPS treatment (Fig. 6a, b). However, suramin attenuated mitochondrial ROS overloading, and this effect was nullified by Ani (Fig. 6a, b). As a consequence of mitochondrial ROS overproduction, the concentrations of cellular antioxidants such as GPX, SOD and GSH were also decreased after exposure to the LPS stimulus (Fig. 6c-e). Interestingly, the suramin treatment reversed the changes in GPX, SOD and GSH levels in hepatocytes (Fig. 6c-e); this effect depended on the JNK pathway because activation of JNK signaling abolished suramin-mediated increases in antioxidant levels. Moreover, caspase- 9 activity, a hallmark of the mitochondrial apoptosis pathway, was also increased by LPS and was reduced to near-normal levels by the suramin treatment (Fig. 6f). However, the Ani treatment re-activated caspase-9 in suramin-treated cells, indicating that the JNK signaling pathway is involved in mitochondrial stress in the setting of LPS-induced inflammatory injury in hepatocytes (Fig. 6f). Additionally, cell viability, as assessed using the
LDH release assay, was reduced by the LPS treatment and was reversed to near-normal levels by suramin supplementation (Fig. 6g). However, activation of the JNK pathway abolished the pro-survival effect of suramin on LPS-treated hepatocytes (Fig. 6g). Similar results were also obtained using the TUNEL assay (Fig. 6h, i). Altogether, this information verified the necessary role of the JNK signaling pathway in mitochondrial stress and hepatocyte death in the context of LPS-induced inflammatory stress.

\section{Discussion}

Inflammation is associated with excessive hepatocyte death during the development of ALF through a poorly understood mechanism. In the present study, inflammation-mediated hepatocyte death resulted from mitochondrial stress, including disrupted mitochondrial bioenergetics, altered mitochondrial dynamics and the activation of the mitochondrial apoptosis pathway. We further verified that increased Mst1 expression was responsible for inflammation-mediated mitochondrial damage. More importantly, we also provided evidence that suramin supplementation represents a protective tool to substantially reduce inflammation-mediated hepatocyte death, and this beneficial mechanism is associated with mitochondrial protection via the JNK-Mst1 signaling pathway. Therefore, our data identify Mst1 upregulation 
A
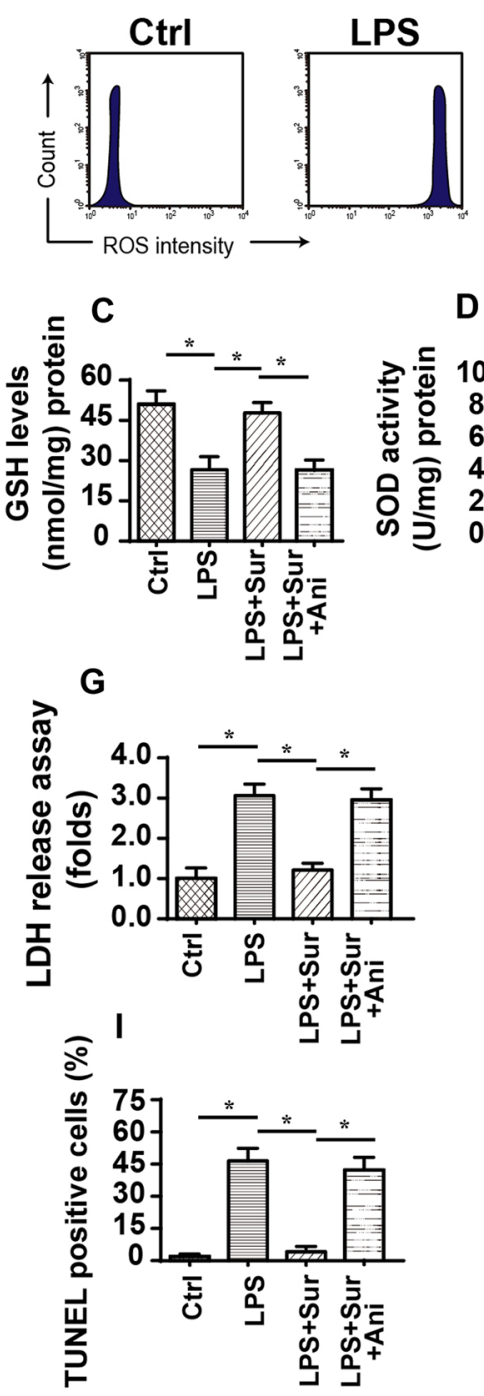

D

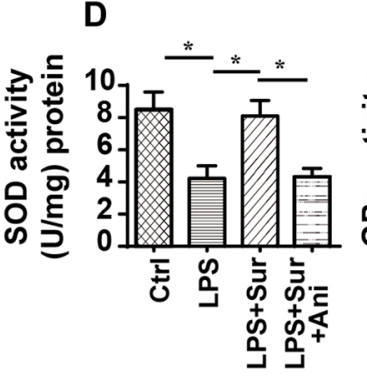

H

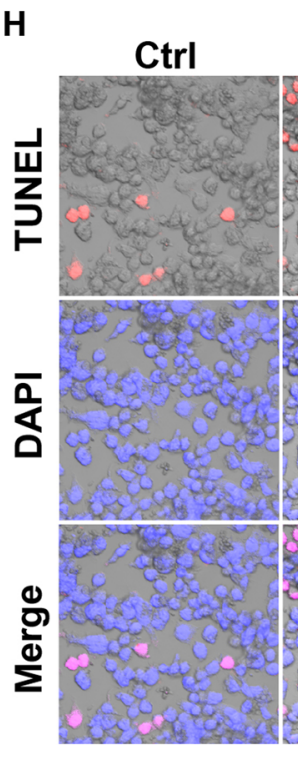

LPS+Sur +Ani
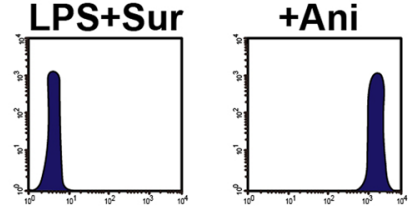

E
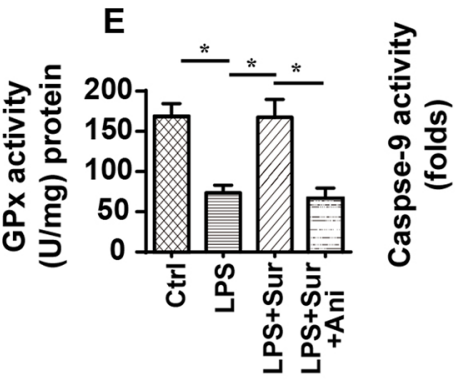

$\mathbf{F}$

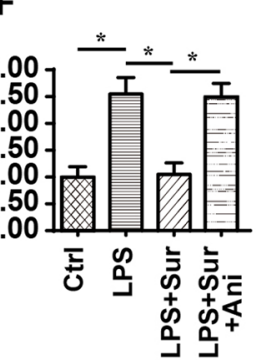

LPS+Sur

LPS

LPS+Sur

+ Ani
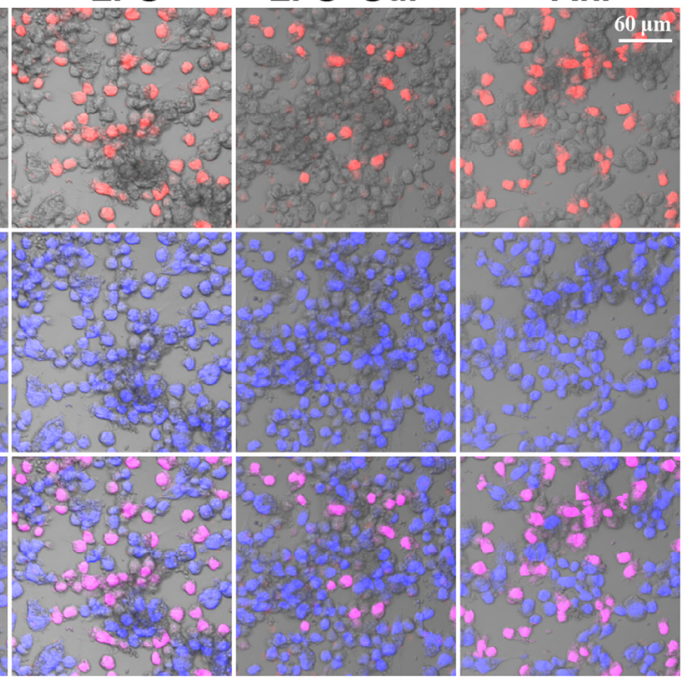

Fig. 6 The JNK pathway is involved in LPS-mediated hepatocyte death and mitochondrial stress. a, b Mitochondrial ROS production was determined using flow cytometry. c-e ELISAs were used to confirm the concentrations of GSH, SOD and GPX in response to JNK activator. f Caspase-9 activity was determined using an ELISA. g Hepatocyte viability was observed using the LDH release assay in cells treated with the JNK activator. $\mathbf{h}, \mathbf{i}$ The TUNEL assay was used to observe the number of apoptotic cells. ${ }^{*} p<0.05$ and subsequent mitochondrial stress as the primary pathogenesis of inflammation-mediated hepatocyte death. On the other hand, we provide an effective and practical way to repress inflammation-mediated mitochondrial stress and hepatocyte damage. However, further studies are required to collect more clinical data to support our findings in the future.

Mitochondria have been defined as a primary target of inflammatory injury. In patients with neuroinflammatory diseases, such as Parkinson's disease, mitochondrial oxidative phosphorylation arrest and mitochondrial ROS overproduction are observed. In mice with high-fat-dietinduced cardiomyopathy, mitochondrial ATP production is reduced, followed by cardiomyocyte death and heart failure. Additionally, in macrophages subjected to inflammatory stress, the JNK pathway is activated and contributes to the initiation of mitochondrial apoptosis. In the murine model of septic encephalopathy, the mitochondria liberate HtrA2/Omi into the cytoplasm, where HtrA2/Omi amplify the cellular oxidative response, brain edema, and neuron death [48]. 
Mechanistically, Mst1 upregulation seems to be the initial signal for mitochondrial dysfunction. Inhibition of Mst1 by suramin supplementation reversed the changes in mitochondrial bioenergetics, sustained mitochondrial dynamics and blocked mitochondrial apoptosis. These results are similar to previous findings. At the molecular level, Mst1 is associated with mitochondrial apoptosis. For example, increased Mst1 has been acknowledged as a primary pathogenesis of diabetic cardiomyopathy. Besides, the activity of mitochondrial anti-apoptotic protein Bcl-2 is also controlled by Mst1 [49]. Moreover, mitochondrial biogenesis, mitochondrial function, and the mitochondrial radical scavenging system are all regulated by Mst1 in the progression of endometriosis [50]. Thus, Mst1 seems to be the upstream mediator of mitochondrial homeostasis. Notably, previous studies have mainly focused on the influence of Mst1 on regulating the activity of the immune response [51], but the actions of Mst1 on inflammation-challenged mitochondria have received little attention. Our study provides evidence supporting the causal effects of Mst1 on mitochondrial dysfunction in the context of inflammatory stress.

In the present study, we used suramin to attenuate mitochondrial damage and inflammatory stress. Our data confirmed that suramin reduced Mst1 expression by inactivating JNK. A previous study [52] demonstrated that suramin inhibits death receptor-induced apoptosis by inactivation of the death-inducing signaling complex (DISC). DISC is known to activate the JNK signaling pathway. Also, it is known that JNK ensures the efficient execution of apoptosis through the coordinated regulation of mitochondrial events and apoptotic gene transcription [53]. Therefore, the repression of the JNK-Mst1 signaling pathway and mitochondrial damage by suramin could be a consequence of DISC inactivation. The anti-inflammatory and anti-apoptotic effects of suramin on liver tissue have been extensively explored in subjects with partial liver grafts [15], liver cirrhosis, carbon tetrachloride-related liver damage, and hepatocellular carcinoma [54]. However, no study has investigated the beneficial effects and protective mechanism of suramin on inflammation-mediated hepatocyte death. Our data provide evidence supporting these functions. Hepatocytes subjected to inflammatory stress were protected by suramin; suramin decreased cell death, sustained mitochondrial function and inactivated the JNK-Mst1 signaling pathway. These data improve our understanding of the hepatoprotective effects of suramin. However, our study was primarily performed in vitro, and thus animal experiments are required to further validate our findings.

Notably, in the present study, we found that Mst1 was modulated by the JNK pathway under LPS-mediated inflammation injury. This finding was similar to previous studies. For example, during cell death, the JNK pathway has been reported to enhance Mst1-mediated pro-apoptotic signaling through phosphorylation at serine 82 [55]. However, several studies identify Mst1 as an upstream activator of the JNK pathway in cardiomyocyte death [56], matrine-mediated liver cancer damage [16], and colorectal cancer apoptosis [57]. These findings confirm the assumption that there is a novel feedback between Mst1 upregulation and JNK pathway activation [55], with implications for our understanding of the signaling mechanism during cell death. Throughout the current study, LPS is used to induce an in vitro model of inflammation-mediated hepatocyte death whereas d-galactosamine/LPS treatment is usually applied to establish a model of ALF. Accordingly, more research is required to support our findings in the development of ALF.

Based on our results, the pathogenesis of inflammationmediated hepatocyte death is associated with mitochondrial dysfunction and activation of the JNK-Mst1 signaling pathway. Suramin supplementation is a very effective method for attenuating inflammation-mediated hepatocyte death and mitochondrial stress. This study identifies the JNK-Mst1mitochondria axis as a novel candidate target for ALF treatments. More importantly, suramin represents an adjuvant treatment to help the liver resist inflammatory stress, with potential implications for its use in the clinic as a remedy for patients with ALF.

Author contributions AZW, JLW, and JW made substantial contributions to the concept and design of the present study, XJD and YZ contributed to the performance of experiments, data analysis and interpretation, and manuscript writing.

Funding This work was supported by Shanghai University of Medicine $\&$ Health Sciences Seed Project (SFP-18-22-14-011).

\section{Compliance with ethical standards}

Conflict of interests The authors declare that they have no competing interests.

Ethical approval Not applicable.

Informed consent Not applicable.

Data availability The datasets used and/or analyzed during the current study are available from the corresponding author on reasonable request.

\section{References}

1. Bikfalvi A (2017) History and conceptual developments in vascular biology and angiogenesis research: a personal view. Angiogenesis 20:463-478

2. Jin Q, Li R, Hu N, Xin T, Zhu P, Hu S, Ma S, Zhu H, Ren J, Zhou $\mathrm{H}$ (2018) DUSP1 alleviates cardiac ischemia/reperfusion injury by suppressing the Mff-required mitochondrial fission and Bnip3related mitophagy via the JNK pathways. Redox Biol 14:576-587 
3. Szabo G, Petrasek J (2015) Inflammasome activation and function in liver disease. Nat Rev Gastroenterol Hepatol 12:387-400

4. Li R, Xin T, Li D, Wang C, Zhu H, Zhou H (2018) Therapeutic effect of sirtuin 3 on ameliorating nonalcoholic fatty liver disease: the role of the ERK-CREB pathway and Bnip3-mediated mitophagy. Redox Biol 18:229-243

5. Bak DH, Na J, Choi MJ, Lee BC, Oh CT, Kim JY, Han HJ, Kim MJ, Kim TH, Kim BJ (2018) Antiapoptotic effects of human placental hydrolysate against hepatocyte toxicity in vivo and in vitro. Int J Mol Med 42:2569-2583

6. Bird TG, Muller M, Boulter L, Vincent DF, Ridgway RA, LopezGuadamillas E, Lu WY, Jamieson T, Govaere O, Campbell AD, Ferreira-Gonzalez S, Cole AM, Hay T, Simpson KJ, Clark W, Hedley A, Clarke M, Gentaz P, Nixon C, Bryce S, Kiourtis C, Sprangers J, Nibbs RJB, Van Rooijen N, Bartholin L, Mcgreal SR, Apte U, Barry ST, Iredale JP, Clarke AR, Serrano M, Roskams TA, Sansom OJ, Forbes SJ (2018) TGFbeta inhibition restores a regenerative response in acute liver injury by suppressing paracrine senescence. Sci Transl Med. 10:eaan1230

7. Zhou H, Du W, Li Y, Shi C, Hu N, Ma S, Wang W, Ren J (2018) Effects of melatonin on fatty liver disease: the role of NR4A1/ DNA-PKcs/p53 pathway, mitochondrial fission, and mitophagy. J Pineal Res 64:e12450

8. Karwi QG, Bice JS, Baxter GF (2017) Pre- and postconditioning the heart with hydrogen sulfide $(\mathrm{H} 2 \mathrm{~S})$ against ischemia/reperfusion injury in vivo: a systematic review and meta-analysis. Basic Res Cardiol 113:6

9. Chen LY, Renn TY, Liao WC, Mai FD, Ho YJ, Hsiao G, Lee AW, Chang HM (2017) Melatonin successfully rescues hippocampal bioenergetics and improves cognitive function following drug intoxication by promoting Nrf2-ARE signaling activity. J Pineal Res 63:e12417

10. Chen DQ, Cao G, Chen H, Liu D, Su W, Yu XY, Vaziri ND, Liu XH, Bai X, Zhang L, Zhao YY (2017) Gene and protein expressions and metabolomics exhibit activated redox signaling and wnt/ beta-catenin pathway are associated with metabolite dysfunction in patients with chronic kidney disease. Redox Biol 12:505-521

11. Galano A, Reiter RJ (2018) Melatonin and its metabolites vs oxidative stress: from individual actions to collective protection. J Pineal Res 65:e12514

12. Souza LEB, Beckenkamp LR, Sobral LM, Fantacini DMC, Melo FUF, Borges JS, Leopoldino AM, Kashima S, Covas DT (2018) Pre-culture in endothelial growth medium enhances the angiogenic properties of adipose-derived stem/stromal cells. Angiogenesis 21:15-22

13. Xiong C, Liu N, Fang L, Zhuang S, Yan H (2014) Suramin inhibits the development and progression of peritoneal fibrosis. J Pharmacol Exp Ther 351:373-382

14. Korrapati MC, Howell LA, Shaner BE, Megyesi JK, Siskind LJ, Schnellmann RG (2013) Suramin: a potential therapy for diabetic nephropathy. PLoS One 8:e73655

15. He S, Rehman H, Shi Y, Krishnasamy Y, Lemasters JJ, Schnellmann RG, Zhong Z (2013) Suramin decreases injury and improves regeneration of ethanol-induced steatotic partial liver grafts. J Pharmacol Exp Ther 344:417-425

16. Reddy KRK, Dasari C, Duscharla D, Supriya B, Ram NS, Surekha MV, Kumar JM, Ummanni R (2018) Dimethylarginine dimethylaminohydrolase-1 (DDAH1) is frequently upregulated in prostate cancer, and its overexpression conveys tumor growth and angiogenesis by metabolizing asymmetric dimethylarginine (ADMA). Angiogenesis 21:79-94

17. Loforese G, Malinka T, Keogh A, Baier F, Simillion C, Montani M, Halazonetis TD, Candinas D, Stroka D (2017) Impaired liver regeneration in aged mice can be rescued by silencing Hippo core kinases MST1 and MST2. EMBO Mol Med 9:46-60
18. Geng C, Zhang Y, Gao Y, Tao W, Zhang H, Liu X, Fang F, Chang Y (2016) Mst1 regulates hepatic lipid metabolism by inhibiting Sirt1 ubiquitination in mice. Biochem Biophys Res Commun 471:444-449

19. Ding M, Ning J, Feng N, Li Z, Liu Z, Wang Y, Wang Y, Li X, Huo C, Jia X, Xu R, Fu F, Wang X, Pei J (2018) Dynamin-related protein 1-mediated mitochondrial fission contributes to posttraumatic cardiac dysfunction in rats and the protective effect of melatonin. J Pineal Res 64:e12447

20. Zhou H, Shi C, Hu S, Zhu H, Ren J, Chen Y (2018) BI1 is associated with microvascular protection in cardiac ischemia reperfusion injury via repressing Syk-Nox2-Drp1-mitochondrial fission pathways. Angiogenesis 21:599-615

21. Van Beijnum JR, Nowak-Sliwinska P, Van Berkel M, Wong TJ, Griffioen AW (2017) A genomic screen for angiosuppressor genes in the tumor endothelium identifies a multifaceted angiostatic role for bromodomain containing 7 (BRD7). Angiogenesis 20:641-654

22. Guers JJ, Zhang J, Campbell SC, Oydanich M, Vatner DE, Vatner SF (2017) Disruption of adenylyl cyclase type 5 mimics exercise training. Basic Res Cardiol 112:59

23. Zhou H, Wang J, Zhu P, Zhu H, Toan S, Hu S, Ren J, Chen Y (2018) NR4A1 aggravates the cardiac microvascular ischemia reperfusion injury through suppressing FUNDC1-mediated mitophagy and promoting Mff-required mitochondrial fission by CK2alpha. Basic Res Cardiol 113:23

24. Choi GH, Lee HY, Back K (2017) Chloroplast overexpression of rice caffeic acid O-methyltransferase increases melatonin production in chloroplasts via the 5-methoxytryptamine pathway in transgenic rice plants. J Pineal Res 63:e12412

25. Blackburn NJR, Vulesevic B, Mcneill B, Cimenci CE, Ahmadi A, Gonzalez-Gomez M, Ostojic A, Zhong Z, Brownlee M, Beisswenger PJ, Milne RW, Suuronen EJ (2017) Methylglyoxal-derived advanced glycation end products contribute to negative cardiac remodeling and dysfunction post-myocardial infarction. Basic Res Cardiol 112:57

26. Zhou H, Wang J, Zhu P, Hu S, Ren J (2018) Ripk3 regulates cardiac microvascular reperfusion injury: the role of IP3R-dependent calcium overload, XO-mediated oxidative stress and F-action/ filopodia-based cellular migration. Cell Signal 45:12-22

27. Peterson YK, Nasarre P, Bonilla IV, Hilliard E, Samples J, Morinelli TA, Hill EG, Klauber-Demore N (2017) Frizzled-5: a high affinity receptor for secreted frizzled-related protein-2 activation of nuclear factor of activated T-cells $\mathrm{c} 3$ signaling to promote angiogenesis. Angiogenesis 20:615-628

28. Hooshdaran B, Kolpakov MA, Guo X, Miller SA, Wang T, Tilley DG, Rafiq K, Sabri A (2017) Dual inhibition of cathepsin G and chymase reduces myocyte death and improves cardiac remodeling after myocardial ischemia reperfusion injury. Basic Res Cardiol 112:62

29. Kleinbongard P, Skyschally A, Gent S, Pesch M, Heusch G (2017) STAT3 as a common signal of ischemic conditioning: a lesson on "rigor and reproducibility" in preclinical studies on cardioprotection. Basic Res Cardiol 113:3

30. Zhou H, Wang J, Hu S, Zhu H, Toanc S, Ren J (2019) BI1 alleviates cardiac microvascular ischemia-reperfusion injury via modifying mitochondrial fission and inhibiting XO/ROS/F-actin pathways. J Cell Physiol 234:5056-5069

31. Schluter KD, Wolf A, Weber M, Schreckenberg R, Schulz R (2017) Oxidized low-density lipoprotein (oxLDL) affects loadfree cell shortening of cardiomyocytes in a proprotein convertase subtilisin/kexin 9 (PCSK9)-dependent way. Basic Res Cardiol 112:63

32. Tenreiro MM, Correia ML, Brito MA (2017) Endothelial progenitor cells in multiple myeloma neovascularization: a brick to the wall. Angiogenesis 20:443-462 
33. Landry NM, Cohen S, Dixon IMC (2017) Periostin in cardiovascular disease and development: a tale of two distinct roles. Basic Res Cardiol 113:1

34. Zhou H, Yue Y, Wang J, Ma Q, Chen Y (2018) Melatonin therapy for diabetic cardiomyopathy: a mechanism involving Syk-mitochondrial complex I-SERCA pathway. Cell Signal 47:88-100

35. Zhou H, Zhu P, Wang J, Zhu H, Ren J, Chen Y (2018) Pathogenesis of cardiac ischemia reperfusion injury is associated with CK2alpha-disturbed mitochondrial homeostasis via suppression of FUNDC1-related mitophagy. Cell Death Differ 25:1080-1093

36. Cortese-Krott MM, Mergia E, Kramer CM, Luckstadt W, Yang J, Wolff G, Panknin C, Bracht T, Sitek B, Pernow J, Stasch JP, Feelisch M, Koesling D, Kelm M (2018) Identification of a soluble guanylate cyclase in RBCs: preserved activity in patients with coronary artery disease. Redox Biol 14:328-337

37. Zhou H, Hu S, Jin Q, Shi C, Zhang Y, Zhu P, Ma Q, Tian F, Chen $Y$ (2017) Mff-dependent mitochondrial fission contributes to the pathogenesis of cardiac microvasculature ischemia/reperfusion injury via induction of mROS-mediated cardiolipin oxidation and HK2/VDAC1 disassociation-involved mPTP opening. J Am Heart Assoc 6:e005328

38. Zhou H, Zhang Y, Hu S, Shi C, Zhu P, Ma Q, Jin Q, Cao F, Tian F, Chen Y (2017) Melatonin protects cardiac microvasculature against ischemia/reperfusion injury via suppression of mitochondrial fission-VDAC1-HK2-mPTP-mitophagy axis. J Pineal Res 63:e12413

39. Zhou H, Li D, Zhu P, Hu S, Hu N, Ma S, Zhang Y, Han T, Ren J, Cao F, Chen Y (2017) Melatonin suppresses platelet activation and function against cardiac ischemia/reperfusion injury via PPARgamma/FUNDC1/mitophagy pathways. J Pineal Res 63:e12438

40. Zhu P, Hu S, Jin Q, Li D, Tian F, Toan S, Li Y, Zhou H, Chen Y (2018) Ripk3 promotes ER stress-induced necroptosis in cardiac IR injury: a mechanism involving calcium overload/XO/ROS/ mPTP pathway. Redox Biol 16:157-168

41. Zhou H, Zhu P, Guo J, Hu N, Wang S, Li D, Hu S, Ren J, Cao F, Chen Y (2017) Ripk3 induces mitochondrial apoptosis via inhibition of FUNDC1 mitophagy in cardiac IR injury. Redox Biol 13:498-507

42. Zhou H, Ma Q, Zhu P, Ren J, Reiter RJ, Chen Y (2018) Protective role of melatonin in cardiac ischemia-reperfusion injury: from pathogenesis to targeted therapy. J Pineal Res 64:e12471

43. Zhou H, Li D, Zhu P, Ma Q, Toan S, Wang J, Hu S, Chen Y, Zhang Y (2018) Inhibitory effect of melatonin on necroptosis via repressing the Ripk3-PGAM5-CypD-mPTP pathway attenuates cardiac microvascular ischemia-reperfusion injury. J Pineal Res 65:e12503

44. Shi C, Cai Y, Li Y, Li Y, Hu N, Ma S, Hu S, Zhu P, Wang W, Zhou $\mathrm{H}$ (2018) Yap promotes hepatocellular carcinoma metastasis and mobilization via governing cofilin/F-actin/lamellipodium axis by regulation of JNK/Bnip3/SERCA/CaMKII pathways. Redox Biol 14:59-71

45. Zhou H, Wang S, Zhu P, Hu S, Chen Y, Ren J (2018) Empagliflozin rescues diabetic myocardial microvascular injury via
AMPK-mediated inhibition of mitochondrial fission. Redox Biol $15: 335-346$

46. Wang X, Song Q (2018) Mst1 regulates post-infarction cardiac injury through the JNK-Drp1-mitochondrial fission pathway. Cell Mol Biol Lett 23:21

47. Li Q, Qi F, Meng X, Zhu C, Gao Y (2018) Mst1 regulates colorectal cancer stress response via inhibiting Bnip3-related mitophagy by activation of JNK/p53 pathway. Cell Biol Toxicol 34:263-277

48. Wang P, Hu Y, Yao D, Li Y (2018) Omi/HtrA2 regulates a mitochondria-dependent apoptotic pathway in a murine model of septic encephalopathy. Cell Physiol Biochem 49:2163-2173

49. Casadonte L, Verhoeff BJ, Piek JJ, Vanbavel E, Spaan JAE, Siebes $M$ (2017) Influence of increased heart rate and aortic pressure on resting indices of functional coronary stenosis severity. Basic Res Cardiol 112:61

50. Zhao Q, Ye M, Yang W, Wang M, Li M, Gu C, Zhao L, Zhang Z, Han W, Fan W, Meng Y (2018) Effect of Mst1 on endometriosis apoptosis and migration: role of Drp1-related mitochondrial fission and parkin-required mitophagy. Cell Physiol Biochem 45:1172-1190

51. Kurz AR, Pruenster M, Rohwedder I, Ramadass M, Schafer K, Harrison U, Gouveia G, Nussbaum C, Immler R, Wiessner JR, Margraf A, Lim DS, Walzog B, Dietzel S, Moser M, Klein C, Vestweber D, Haas R, Catz SD, Sperandio M (2016) MST1dependent vesicle trafficking regulates neutrophil transmigration through the vascular basement membrane. J Clin Invest 126:4125-4139

52. Sajib S, Zahra FT, Lionakis MS, German NA, Mikelis CM (2018) Mechanisms of angiogenesis in microbe-regulated inflammatory and neoplastic conditions. Angiogenesis 21:1-14

53. Dhanasekaran DN, Reddy EP (2008) JNK signaling in apoptosis. Oncogene 27:6245-6251

54. Ghiroldi A, Piccoli M, Ciconte G, Pappone C, Anastasia L (2017) Regenerating the human heart: direct reprogramming strategies and their current limitations. Basic Res Cardiol 112:68

55. Fernandez Vazquez G, Reiter RJ, Agil A (2018) Melatonin increases brown adipose tissue mass and function in Zucker diabetic fatty rats: implications for obesity control. J Pineal Res 64:e12472

56. Cheng Z, Zhang M, Hu J, Lin J, Feng X, Wang S, Wang T, Gao E, Wang H, Sun D (2019) Cardiac-specific Mst1 deficiency inhibits ROS-mediated JNK signalling to alleviate Ang II-induced cardiomyocyte apoptosis. J Cell Mol Med 23:543-555

57. Kalyanaraman B, Cheng G, Hardy M, Ouari O, Lopez M, Joseph J, Zielonka J, Dwinell MB (2018) A review of the basics of mitochondrial bioenergetics, metabolism, and related signaling pathways in cancer cells: Therapeutic targeting of tumor mitochondria with lipophilic cationic compounds. Redox Biol 14:316-327

Publisher's Note Springer Nature remains neutral with regard to jurisdictional claims in published maps and institutional affiliations. 